\title{
Multiplicity of Positive Solutions for a Class of Inhomogeneous Neumann Problems Involving the $p(x)$-Laplacian
}

Xianling Fan and Shao-Gao Deng

\begin{abstract}
We study the existence and multiplicity of positive solutions for the inhomogeneous Neumann boundary value problems involving the $p(x)$ Laplacian of the form

$$
\left\{\begin{array}{l}
-\operatorname{div}\left(|\nabla u|^{p(x)-2} \nabla u\right)+\lambda|u|^{p(x)-2} u=f(x, u) \text { in } \Omega \\
|\nabla u|^{p(x)-2} \frac{\partial u}{\partial \eta}=\varphi \text { on } \partial \Omega,
\end{array}\right.
$$

where $\Omega$ is a bounded smooth domain in $\mathbf{R}^{N}, p \in C^{1}(\bar{\Omega})$ and $p(x)>1$ for $x \in \bar{\Omega}, \varphi \in C^{0, \gamma}(\partial \Omega)$ with $\gamma \in(0,1), \varphi \geq 0$ and $\varphi \not \equiv 0$ on $\partial \Omega$. Using the sub-supersolution method and the variational method, under appropriate assumptions on $f$, we prove that, there exists $\lambda_{*}>0$ such that the problem has at least two positive solutions if $\lambda>\lambda_{*}$, has at least one positive solution if $\lambda=\lambda_{*}$, and has no positive solution if $\lambda<\lambda_{*}$. To prove the result we establish a special strong comparison principle for the Neumann problems.
\end{abstract}

Mathematics Subject Classification (2000). 35J65, 35J70, 35J20.

Keywords. $p(x)$-Laplacian equation; Neumann problem; positive solution; sub-supersolution method; variational method.

\section{Introduction}

The purpose of this paper is to study the existence and multiplicity of positive solutions for the following inhomogeneous Neumann problem involving the $p(x)$ Laplacian

$$
\left\{\begin{array}{l}
-\operatorname{div}\left(|\nabla u|^{p(x)-2} \nabla u\right)+\lambda|u|^{p(x)-2} u=f(x, u) \text { in } \Omega \\
|\nabla u|^{p(x)-2} \frac{\partial u}{\partial \eta}=\varphi \text { on } \partial \Omega
\end{array}\right.
$$

The research was supported by the National Natural Science Foundation of China $(10371052,10671084)$. 
where $\Omega$ is a bounded smooth domain in $\mathbf{R}^{N}$ with $C^{1, \beta}$ smooth boundary, $\eta$ is the unit outward normal to $\partial \Omega, \lambda \in \mathbf{R}, p, f$ and $\varphi$ satisfy the following assumption $(A)$ :

Assumption $(A) . p \in C^{1}(\bar{\Omega}), p_{-}:=\inf _{\bar{\Omega}} p(x)>1, f \in C(\bar{\Omega} \times \mathbf{R}), \varphi \in C^{0, \gamma}(\partial \Omega)$ with some $\gamma \in(0,1), \varphi \geq 0$ and $\varphi \neq \equiv 0$ on $\partial \Omega$.

The operator $-\Delta_{p(x)} u:=-\operatorname{div}\left(|\nabla u|^{p(x)-2} \nabla u\right)$ is called $p(x)$-Laplacian, which becomes $p$-Laplacian when $p(x) \equiv p$ (a constant). The study of various mathematical problems with variable exponent has been received considerable attention in recent years. These problems are interesting in applications (see e.g. [37,42] and raise many difficult mathematical problems. We refer to $[14,38]$ for the overview and references of this subject, and to [2,4,16-20,22,31-33,41] for the study of the $p(x)$-Laplacian equations and the corresponding variational problems.

Many authors have studied the inhomogeneous (and nonlinear) Neumann problems involving the $p$-Laplacian, see e.g. $[1,13,26,34,35,40]$ and the references therein. In [13] and [1] the authors have studied the problem $\left(1.1_{\lambda}\right)$ in the cases of $p(x) \equiv p=2$ and of $p(x) \equiv p>1$ respectively. The aim of the present paper is to generalize the main results of $[1,13]$ to the $p(x)$-Laplacian case. In $[1,13]$ only the case that $f(x, u)=|u|^{q-2} u$ is considered, where $p<q \leq p^{*}$. In the present paper the function $f(x, u)$ is of general type which includes $|u|^{q(x)-2} u$ as a special case. In studying the existence of positive solutions of $\left(1.1_{\lambda}\right)$ we do not restrict the growth condition of $f(x, u)$, but in studying the multiplicity of positive solutions of $\left(1.1_{\lambda}\right)$, in this paper, we shall restrict ourselves to the case that $f(x, u)$ satisfies the subcritical growth condition because the study of the $p(x)$-Laplacian equations in the critical growth case is very difficult and requires some special preliminaries which are not ready up to the present.

In this paper we use the following notations:

$$
\begin{aligned}
& p_{-}=\inf _{\bar{\Omega}} p(x), \quad p_{+}=\sup _{\bar{\Omega}} p(x), \\
& p^{*}(x):=\left\{\begin{array}{cl}
\frac{N p(x)}{N-p(x)}, & \text { if } p(x)<N, \\
\infty, & \text { if } p(x) \geq N,
\end{array}\right. \\
& F(x, t)=\int_{0}^{t} f(x, s) d s, \\
& \Lambda=\left\{\lambda \in \mathbf{R} \text { : there exists at least a positive solution of }\left(1.1_{\lambda}\right)\right\}, \\
& \lambda_{*}=\inf \Lambda \text {. }
\end{aligned}
$$

The main results of this paper are the following theorems in which we always suppose that the Assumption $(A)$ holds.

Theorem 1.1. Suppose that $f$ satisfies the following conditions:

$$
f(x, t) \geq 0, \quad \forall x \in \Omega, \quad \forall t \geq 0,
$$


and

for each $x \in \Omega, f(x, t)$ is nondecreasing with respect to $t \geq 0$.

Then $\Lambda \neq \emptyset, \lambda_{*} \geq 0$ and $\left(\lambda_{*},+\infty\right) \subset \Lambda$. Moreover, for every $\lambda>\lambda_{*}$, there exists a minimal positive solution $u_{\lambda}$ of $\left(1.1_{\lambda}\right)$ such that $u_{\lambda_{1}} \leq u_{\lambda_{2}}$ if $\lambda_{*}<\lambda_{2}<\lambda_{1}$.

Theorem 1.2. Suppose that (1.2) and (1.3) hold, and there exist positive constants $M, c_{1}$ and $c_{2}$ such that

$$
f(x, t) \leq c_{1}+c_{2} t^{q(x)-1}, \quad \forall x \in \Omega, \quad \forall t \geq M,
$$

where $q \in C(\bar{\Omega})$ and $1 \leq q(x)<p^{*}(x)$ for $x \in \bar{\Omega}$. Then for each $\lambda \in\left(\lambda_{*},+\infty\right)$, $\left(1.1_{\lambda}\right)$ has a positive solution $u_{\lambda}$ which is a local minimizer of the energy functional associated with $\left(1.1_{\lambda}\right)$. Moreover, for $\lambda_{*}<\lambda_{2}<\lambda_{1}$, there are the corresponding local minimizers $u_{\lambda_{1}}$ and $u_{\lambda_{2}}$ such that $u_{\lambda_{1}}<u_{\lambda_{2}}$ on $\bar{\Omega}$.

Theorem 1.3. Under the assumptions of Theorem 1.2, also suppose that there exist positive constants $M$ and $\theta>p_{+}$such that

$$
0<\theta F(x, t) \leq f(x, t), \quad \forall x \in \Omega, \quad \forall t \geq M .
$$

Then for each $\lambda \in\left(\lambda_{*},+\infty\right),\left(1.1_{\lambda}\right)$ has at least two positive solutions $u_{\lambda}$ and $v_{\lambda}$, where $u_{\lambda}$ is a local minimizer of the energy functional and $u_{\lambda} \leq v_{\lambda}$.

\section{Theorem 1.4.}

(1) Suppose that $f$ satisfies (1.2),

$$
f(x, 0) \leq f(x, t) \quad \text { for } t>0 \text { and } \quad x \in \Omega,
$$

and the following conditions:

$$
f(x, t) \leq c_{1}+c_{2} t^{r(x)-1}, \quad \forall x \in \Omega, \quad \forall t \geq M,
$$

where $M, c_{1}$ and $c_{2}$ are positive constants, $r \in C(\bar{\Omega})$ and $1 \leq r(x)<p(x)$ for $x \in \bar{\Omega}$. Then $\lambda_{*}=0$.

(2) If $f$ satisfies (1.2)-(1.5), then $\lambda_{*}>0$ and $\lambda_{*} \in \Lambda$.

The underlying idea for proving Theorems 1.1-1.4 is similar to the one of [1] The special features of this class of problems considered in the present paper are that they involve the variable exponent. The $p(x)$-Laplacian possesses more complicated nonlinearities than the $p$-Laplacian. For example, it is inhomogeneous. To prove Theorems 1.1-1.4 we use the theory of variable exponent Sobolev spaces, established first by Kováčik and Rákosník [27], and some research results obtained recently for the $p(x)$-Laplacian equations. In particular we use the results of [18] on the global $C^{1, \alpha}$ regularity of the weak solutions for the $p(x)$-Laplacian equations. The main method used in this paper is the sub-supersolution method for the Neumann problems involving the $p(x)$-Laplacian, which is similar to that given in [17] for the Dirichlet problems involving the $p(x)$-Laplacian. A main difficulty for proving Theorem 1.2 is that a special strong comparison principle is required. It is well known that, when $p \neq 2$, the strong comparison principles for the $p$-Laplacian equations are very complicated (see e.g. $[11,12,23,36])$. In $[17,24,25]$ the required 
strong comparison principles for the Dirichlet problems have be established, however, they cannot be applied to the Neumann problems. To prove Theorem 1.2, we establish a special strong comparison principle for the Neumann problem $\left(1.1_{\lambda}\right)$ (see Lemma 3.6 in Section 3), which is also valid for the homogeneous Neumann boundary value problems.

In Section 2 we give some preliminary knowledge. In Section 3 we give the proof of Theorems 1.1-1.4.

\section{Preliminaries}

Let $\Omega$ be a bounded smooth domain in $\mathbf{R}^{N}, p \in L^{\infty}(\Omega)$ and $p_{-} \geq 1$. Denote by $\mathbf{S}(\Omega)$ the set of all measurable real functions defined on $\Omega$. Note that two measurable functions are considered as the same element of $\mathbf{S}(\Omega)$ when they are equal almost everywhere. Define the variable exponent Lebesgue space $L^{p(x)}(\Omega)$ by

with the norm

$$
L^{p(x)}(\Omega)=\left\{u \in \mathbf{S}(\Omega): \int_{\Omega}|u(x)|^{p(x)} d x<\infty\right\}
$$

$$
|u|_{L^{p(x)}(\Omega)}=|u|_{p(x)}=\inf \left\{\sigma>0: \int_{\Omega}\left|\frac{u(x)}{\sigma}\right|^{p(x)} d x \leq 1\right\},
$$

and the variable exponent Sobolev space $W^{1, p(x)}(\Omega)$ by

$$
W^{1, p(x)}(\Omega)=\left\{u \in L^{p(x)}(\Omega):|\nabla u| \in L^{p(x)}(\Omega)\right\}
$$

with the norm

$$
\|u\|=\|u\|_{W^{1, p(x)}(\Omega)}=|u|_{L^{p(x)}(\Omega)}+|\nabla u|_{L^{p(x)}(\Omega)} .
$$

Denote by $W_{0}^{1, p(x)}(\Omega)$ the closure of $C_{0}^{\infty}(\Omega)$ in $W^{1, p(x)}(\Omega)$. The spaces $L^{p(x)}(\Omega)$, $W^{1, p(x)}(\Omega)$ and $W_{0}^{1, p(x)}(\Omega)$ are all separable Banach spaces. When $p_{-}>1$ these spaces are reflexive. We refer to $[14,15,21,27,38,39]$ for the elementary properties of these spaces. The variable exponent Sobolev space $W^{1, p(x)}(\Omega)$ is a generalization of the standard Sobolev space $W^{1, p}(\Omega)$. Another generalization of the standard Sobolev space is the Orlicz-Sobolev space. For the study of quasilinear elliptic equations in Orlicz-Sobolev space setting we refer to [28] and the references therein.

Let $\lambda>0$. Define for $u \in W^{1, p(x)}(\Omega)$,

$$
\|u\|_{\lambda}=\inf \left\{\sigma>0: \int_{\Omega}\left(\left|\frac{\nabla u}{\sigma}\right|^{p(x)}+\lambda\left|\frac{u}{\sigma}\right|^{p(x)}\right) d x \leq 1\right\} .
$$

Then $\|u\|_{\lambda}$ is a norm on $W^{1, p(x)}(\Omega)$ equivalent to $\|u\|_{W^{1, p(x)}(\Omega)}$.

By the definition of $\|u\|_{\lambda}$ we have the following 
Proposition 2.1 (See $[21,27])$. Put $\rho_{\lambda}(u)=\int_{\Omega}\left(|\nabla u|^{p(x)}+\lambda|u|^{p(x)}\right) d x$ for $\lambda>0$ and $u \in W^{1, p(x)}(\Omega)$. We have:
1). $\|u\|_{\lambda} \geq 1 \Longrightarrow\|u\|_{\lambda}^{p_{-}} \leq \rho_{\lambda}(u) \leq\|u\|_{\lambda}^{p_{+}}$
2). $\|u\|_{\lambda} \leq 1 \Longrightarrow\|u\|_{\lambda}^{p_{+}} \leq \rho_{\lambda}(u) \leq\|u\|_{\lambda}^{p_{-}}$;
3). $\left\|u_{k}\right\|_{\lambda} \rightarrow 0 \Longleftrightarrow \rho_{\lambda}\left(u_{k}\right) \rightarrow 0($ as $k \rightarrow \infty)$;
4). $\left\|u_{k}\right\|_{\lambda} \rightarrow \infty \Longleftrightarrow \rho_{\lambda}\left(u_{k}\right) \rightarrow \infty($ as $k \rightarrow \infty)$.

Proposition 2.2 (See $[\mathbf{2 1}])$. Let $p \in C(\bar{\Omega})$. If $q \in C(\bar{\Omega})$ satisfies the condition

$$
1 \leq q(x)<p^{*}(x), \quad \forall x \in \bar{\Omega},
$$

then there is a compact embedding $W^{1, p(x)}(\Omega) \hookrightarrow L^{q(x)}(\Omega)$.

For $A \subset \bar{\Omega}$, denote $p_{-}(A)=\inf \{p(x): x \in A\}$ and $p_{+}(A)=\sup \{p(x): x \in A\}$. Put

$$
p^{\partial}(x):=(p(x))^{\partial}:=\left\{\begin{array}{cl}
\frac{(N-1) p(x)}{N-p(x)}, & \text { if } p(x)<N \\
\infty, & \text { if } p(x) \geq N .
\end{array}\right.
$$

Proposition 2.3. Let $p \in C(\bar{\Omega})$ and $p_{-}>1$. If $q \in C(\partial \Omega)$ satisfies the condition

$$
1 \leq q(x)<p^{\partial}(x), \quad \forall x \in \partial \Omega,
$$

then there is a compact embedding $W^{1, p(x)}(\Omega) \hookrightarrow L^{q(x)}(\partial \Omega)$. In particular, there is a compact embedding $W^{1, p(x)}(\Omega) \hookrightarrow L^{p(x)}(\partial \Omega)$.

Proof. Let $u \in W^{1, p(x)}(\Omega)$. Given any $x \in \partial \Omega$, by (2.2), there is a relatively open neighborhood $\Omega_{x}$ of $x$ in $\bar{\Omega}$ such that

$$
q_{+}\left(\partial \Omega \cap \Omega_{x}\right)<\left(p_{-}\left(\Omega_{x}\right)\right)^{\partial} .
$$

Now $\left.u\right|_{\Omega_{x}} \in W^{1, p(x)}\left(\Omega_{x}\right) \subset W^{1, p_{-}\left(\Omega_{x}\right)}\left(\Omega_{x}\right)$. By Sobolev trace embedding theorem (see e.g. [3]), $W^{1, p_{-}\left(\Omega_{x}\right)}\left(\Omega_{x}\right) \hookrightarrow L^{q_{+}\left(\partial \Omega \cap \Omega_{x}\right)}\left(\partial \Omega \cap \Omega_{x}\right)$, so $\left.u\right|_{\Omega_{x}} \in L^{q_{+}\left(\partial \Omega \cap \Omega_{x}\right)}(\partial \Omega \cap$ $\left.\Omega_{x}\right) \subset L^{q(x)}\left(\partial \Omega \cap \Omega_{x}\right)$. Using the finite covering theorem for the compact set $\partial \Omega$, we can obtain $u \in L^{q(x)}(\partial \Omega)$. This shows that there is an embedding $W^{1, p(x)}(\Omega) \hookrightarrow$ $L^{q(x)}(\partial \Omega)$. Since each embedding $W^{1, p_{-}\left(\Omega_{x}\right)}\left(\Omega_{x}\right) \hookrightarrow L^{q_{+}\left(\partial \Omega \cap \Omega_{x}\right)}\left(\partial \Omega \cap \Omega_{x}\right)$ is compact, we can see that the embedding $W^{1, p(x)}(\Omega) \hookrightarrow L^{q(x)}(\partial \Omega)$ is compact.

Throughout the paper $f$ and $\varphi$ will be supposed to satisfy Assumption $(A)$.

\section{Definition 2.1.}

(1) $u \in W^{1, p(x)}(\Omega)$ is called a weak solution of the problem $\left(1.1_{\lambda}\right)$ if for all $v \in W^{1, p(x)}(\Omega)$,

$$
\int_{\Omega}\left(|\nabla u|^{p(x)-2} \nabla u \nabla v+\lambda|u|^{p(x)-2} u v\right) d x-\int_{\partial \Omega} \varphi v d \sigma=\int_{\Omega} f(x, u) v d x .
$$

(2) $u \in W^{1, p(x)}(\Omega)$ is called a generalized solution of the equation

$$
-\operatorname{div}\left(|\nabla u|^{p(x)-2} \nabla u\right)+\lambda|u|^{p(x)-2} u=f(x, u) \text { in } \Omega,
$$


if for all $v \in C_{0}^{\infty}(\Omega)$

$$
\int_{\Omega}\left(|\nabla u|^{p(x)-2} \nabla u \nabla v+\lambda|u|^{p(x)-2} u v\right) d x=\int_{\Omega} f(x, u) v d x .
$$

Obviously, every weak solution of problem $\left(1.1_{\lambda}\right)$ is also a generalized solution of equation $\left(2.4_{\lambda}\right)$.

In this paper, we need the global regularity results for the weak solution of $\left(1.1_{\lambda}\right)$, which are stated in the following Proposition 2.4. For the $L^{\infty}(\Omega)$ and $C^{0, \alpha}(\bar{\Omega})$ regularity see [20], and for the $C^{1, \alpha}(\bar{\Omega})$ regularity see [18] in which the global $C^{1, \alpha}(\bar{\Omega})$ regularity results for the $p$-Laplacian equations obtained by Lieberman $[29,30]$ are generalized to the case of the $p(x)$-Laplacian equations.

\section{Proposition 2.4.}

(1) (see $[20,18])$ If $p \in C(\bar{\Omega})$ and $f$ satisfy the sub-critical growth condition (1.4), then $u \in L^{\infty}(\Omega)$ for every weak solution $u$ of $\left(1.1_{\lambda}\right)$, and $|u|_{L^{\infty}(\Omega)}$ depends only on $\|u\|_{\lambda},|\varphi|_{L^{\infty}(\partial \Omega)}, p_{-}, p_{+}, N, \lambda, q_{+}, c_{1}$ and $c_{2}$.

(2) (see $[20,18])$ Let $u \in W^{1, p(x)}(\Omega) \cap L^{\infty}(\Omega)$ be a weak solution of $\left(1.1_{\lambda}\right)$. If the function $p$ is log-Hölder continuous on $\bar{\Omega}$, i.e., there is a positive constant $H$ such that

$$
|p(x)-p(y)| \leq \frac{H}{-\log |x-y|} \quad \text { for } \quad x, y \in \bar{\Omega} \quad \text { with } \quad|x-y| \leq \frac{1}{2},
$$

then $u \in C^{0, \alpha}(\bar{\Omega})$ for some $\alpha \in(0,1)$.

(3) (see [18]) If in the statement (2), the condition (2.6) is replaced by that $p$ $\in C^{0, \alpha_{1}}(\bar{\Omega})$, then $u \in C^{1, \alpha}(\bar{\Omega})$ for some $\alpha \in(0,1)$ and $\|u\|_{C^{1, \alpha}(\bar{\Omega})}$ depends only on $|u|_{L^{\infty}(\Omega)},\|\varphi\|_{C^{0, \gamma}(\partial \Omega)},\|p\|_{C^{0, \alpha_{1}}(\bar{\Omega})}, p_{-}, p_{+}, N, \lambda, q_{+}, c_{1}, c_{2}$ and $\Omega$.

For $u, v \in \mathbf{S}(\Omega)$, we write $u \leq v$ if $u(x) \leq v(x)$ for a.e. $x \in \Omega$. Define $u^{+}(x)=\max \{u(x), 0\}$ and $u^{-}(x)=\max \{-u(x), 0\}$.

Proposition 2.5 (See [22]). (A strong maximum principle) Suppose that $p \in C^{1}(\Omega)$, $u$ is a generalized solution of equation $\left(2.4_{\lambda}\right), u \geq 0, u \neq \equiv$ and $f(x, u) \geq 0$ in $\Omega$. Then $u>0$ in $\Omega$. In addition, if $\Omega$ satisfies the interior-ball condition and for some $x_{0} \in \partial \Omega, u\left(x_{0}\right)=0$ and $u \in C^{1}\left(\Omega \cup\left\{x_{0}\right\}\right)$, then $\frac{\partial u}{\partial \eta}\left(x_{0}\right)<0$.

Definition 2.2. $u \in W^{1, p(x)}(\Omega)$ is called a subsolution (resp. supersolution) of $\left(1.1_{\lambda}\right)$ if for all $v \in W^{1, p(x)}(\Omega)$ with $v \geq 0$,

$$
\int_{\Omega}\left(|\nabla u|^{p(x)-2} \nabla u \nabla v+\lambda|u|^{p(x)-2} u v\right) d x-\int_{\partial \Omega} \varphi v d \sigma \leq(\text { resp. } \geq) \int_{\Omega} f(x, u) v d x .
$$

Proposition 2.6 (See [17]). Let $\lambda>0$ and $q \in C(\bar{\Omega})$ satisfy (2.1). Then for each $h \in L^{\frac{q(x)}{q(x)-1}}(\Omega)$, the problem

$$
\left\{\begin{array}{l}
-\operatorname{div}\left(|\nabla u|^{p(x)-2} \nabla u\right)+\lambda|u|^{p(x)-2} u=h(x) \quad \text { in } \Omega \\
|\nabla u|^{p(x)-2} \frac{\partial u}{\partial \eta}=\varphi \text { on } \partial \Omega
\end{array}\right.
$$

has a unique solution $u \in W^{1, p(x)}(\Omega)$. 
Let $q \in C(\bar{\Omega})$ satisfy $(2.1)$. For $h \in L^{\frac{q(x)}{q(x)-1}}(\Omega)$, we denote by $K(h)=K_{\lambda}(h)=$ $u$ the unique solution of $\left(2.7_{\lambda}\right) . K=K_{\lambda}$ is called the solution operator for $\left(2.7_{\lambda}\right)$.

\section{Proposition 2.7.}

(1) (see [17]) The mapping $K: L^{\frac{q(x)}{q(x)-1}}(\Omega) \rightarrow W^{1, p(x)}(\Omega)$ is continuous and bounded. Moreover, the mapping $K: L^{\frac{q(x)}{q(x)-1}}(\Omega) \rightarrow L^{q(x)}(\Omega)$ is completely continuous since the embedding $W^{1, p(x)}(\Omega) \hookrightarrow L^{q(x)}(\Omega)$ is compact.

(2) (see [17]) If p is log-Hölder continuous on $\bar{\Omega}$, then the mapping $K: L^{\infty}(\Omega) \rightarrow$ $C^{0, \alpha}(\bar{\Omega})$ is bounded, and hence the mapping $K: L^{\infty}(\Omega) \rightarrow C(\bar{\Omega})$ is completely continuous.

(3) (see [17]) If $p$ is Hölder continuous on $\bar{\Omega}$, then the mapping $K: L^{\infty}(\Omega) \rightarrow$ $C^{1, \alpha}(\bar{\Omega})$ is bounded, and hence the mapping $K: L^{\infty}(\Omega) \rightarrow C^{1}(\bar{\Omega})$ is completely continuous.

(4) $K$ is an increasing operator, that is, $K\left(h_{1}\right) \leq K\left(h_{2}\right)$ if $h_{1} \leq h_{2}$.

Proposition 2.8. If $h \in L^{\frac{q(x)}{q(x)-1}}(\Omega)$ and $h \geq 0$, where $q \in C(\bar{\Omega})$ satisfy $(2.1)$, then $K(h) \geq 0$. If $p \in C^{1}(\Omega), h \in L^{\infty}(\Omega)$ and $h \geq 0$, then $K(h)>0$ on $\bar{\Omega}$.

Proof. Let $h \geq 0$ and $K(h)=u$. Then $u$ is the unique global minimizer of the energy functional

$$
I_{\lambda}(u)=\int_{\Omega} \frac{|\nabla u|^{p(x)}+\lambda|u|^{p(x)}}{p(x)} d x-\int_{\partial \Omega} \varphi u d \sigma-\int_{\Omega} h u d x, \forall u \in W^{1, p(x)}(\Omega) .
$$

Setting $v(x)=|u(x)|$ we can see that $I_{\lambda}(v) \leq I_{\lambda}(u)$, which shows that $u=v$. Hence $K(h)=u \geq 0$. In the case that $p \in C^{1}(\Omega), h \in L^{\infty}(\Omega)$ and $h \geq 0$, we have $K(h)=u \in C^{1}(\bar{\Omega}), u \geq 0$ and $u \not \equiv 0$. By Proposition 2.5 and noting that $\varphi \geq 0$, we obtain $u>0$ on $\bar{\Omega}$.

In this paper we will use the following sub-supersolution principle, the proof of which is based on the well known fixed point theorem for the increasing operator on the order interval (see e.g. [5]) and is similar to that given in [17] for Dirichlet problems involving the $p(x)$-Laplacian.

Theorem 2.1 (A sub-supersolution principle). Let Assumption (A) hold and $\lambda>0$. Suppose that $u_{0}, v^{0} \in W^{1, p(x)}(\Omega) \cap L^{\infty}(\Omega), u_{0}$ and $v^{0}$ are a subsolution and $a$ supersolution of $\left(1.1_{\lambda}\right)$ respectively, and $u_{0} \leq v^{0}$. If $f$ satisfies the condition:

$$
f(x, t) \text { is nondecreasing in } t \in\left[\inf u_{0}(x), \sup v^{0}(x)\right] \text {, }
$$

then $\left(1.1_{\lambda}\right)$ has a minimal solution $u_{*}$ and a maximal solution $v^{*}$ in the order interval $\left[u_{0}, v^{0}\right]$, i.e., $u_{0} \leq u_{*} \leq v^{*} \leq v^{0}$ and if $u$ is any solution of $\left(1.1_{\lambda}\right)$ such that $u_{0} \leq u \leq v^{0}$, then $u_{*} \leq u \leq v^{*}$. 
The energy functional corresponding to $\left(1.1_{\lambda}\right)$ is

$$
\begin{aligned}
J_{\lambda}(u)= & \int_{\Omega} \frac{|\nabla u|^{p(x)}+\lambda|u|^{p(x)}}{p(x)} d x-\int_{\partial \Omega} \varphi u d \sigma \\
& -\int_{\Omega} F(x, u) d x, \quad \forall u \in W^{1, p(x)}(\Omega) .
\end{aligned}
$$

The critical points of $J_{\lambda}$ are just the solutions of $\left(1.1_{\lambda}\right)$. Many authors, for example, Chang [10], Brezis-Nirenberg [9] and Ambrosetti-Brezis-Cerami [6], have combined the sub-supersolution method with the variational method and studied successfully the semilinear elliptic problems, where a key lemma is that a local minimizer of the associated energy functional in the $C^{1}$-topology is also a local minimizer in the $H^{1}$-topology. Such lemma have been extended to the case of the $p$-Laplacian equations (see $[8,25]$ ) and also to the case of the $p(x)$-Laplacian equations (see [17, Theorem 3.1]). The Theorem 3.1 of [17] concerns the Dirichlet problems, but the method for proving the theorem is also valid for the Neumann problems. Thus we have the following.

Theorem 2.2 (See [17] Theorem 3.1). Let $\lambda>0$ and Assumption (A) and (1.4) hold. If $u \in C^{1}(\bar{\Omega})$ is a local minimizer of $J_{\lambda}$ in the $C^{1}(\bar{\Omega})$-topology, then $u$ is also a local minimizer of $J_{\lambda}$ in the $W^{1, p(x)}(\Omega)$-topology.

\section{Proof of theorems}

In this section we shall prove Theorems 1.1-1.4. We always suppose that the Assumption $(A)$ holds. Since only the positive solutions are considered, without loss of generality, we can assume that

$$
f(x, t)=f(x, 0) \text { for } t<0 \text { and } x \in \bar{\Omega},
$$

otherwise we may replace $f(x, t)$ by $f^{(+)}(x, t)$, where

$$
f^{(+)}(x, t)=\left\{\begin{array}{l}
f(x, t) \text { if } t \geq 0, \\
f(x, 0) \text { if } t<0 .
\end{array}\right.
$$

The proof of Theorem 1.1 consists of the following four Lemmata 3.1-3.4.

Lemma 3.1. Let (1.2) hold. Then $\lambda \notin \Lambda$ if $\lambda \leq 0$

Proof. Let $\lambda \in \Lambda$ and $u$ be a positive solution of $\left(1.1_{\lambda}\right)$. Taking $v \equiv 1$ as a test function in $(2.3)$ yields

$$
\lambda \int_{\Omega} u^{p(x)-1} d x=\int_{\Omega} f(x, u) d x+\int_{\partial \Omega} \varphi d \sigma,
$$

which implies $\lambda>0$ because the value of the right side in (3.1) is positive.

Lemma 3.2. Let (1.2) and (1.3) hold. Then $\Lambda \neq \emptyset$. 
Proof. By Propositions 2.6, 2.8 and 2.4.(3), the problem

$$
\left\{\begin{array}{l}
-\operatorname{div}\left(|\nabla u|^{p(x)-2} \nabla u\right)+|u|^{p(x)-2} u=0 \quad \text { in } \Omega \\
|\nabla u|^{p(x)-2} \frac{\partial u}{\partial \eta}=\varphi \text { on } \partial \Omega,
\end{array}\right.
$$

has a unique positive solution $w_{1} \in C^{1}(\bar{\Omega})$ and $w_{1}(x) \geq \varepsilon>0$ for $x \in \bar{\Omega}$. We can assume $\varepsilon \leq 1$. Put $d=\sup \left\{f\left(x, w_{1}(x)\right): x \in \bar{\Omega}\right\}, M_{1}=\frac{d}{\varepsilon^{p}+-1}$ and $\lambda_{1}=1+M_{1}$. Then

$$
\begin{aligned}
-\Delta_{p(x)} w_{1}+\lambda_{1} w_{1}^{p(x)-1} & =-\Delta_{p(x)} w_{1}+w_{1}^{p(x)-1}+M_{1} w_{1}^{p(x)-1} \\
& \geq M_{1} \varepsilon^{p_{+}-1}=d \geq f\left(x, w_{1}(x)\right) .
\end{aligned}
$$

This shows that $w_{1}$ is a supersolution of the problem $\left(1.1_{\lambda_{1}}\right)$. Obviously 0 is a subsolution of $\left(1.1_{\lambda_{1}}\right)$. By Theorem 2.1, $\left(1.1_{\lambda_{1}}\right)$ has a solution $u_{\lambda_{1}}$ such that $0 \leq$ $u_{\lambda_{1}} \leq w_{1}$. By Proposition 2.8, $u_{\lambda_{1}}>0$ on $\bar{\Omega}$. So $\lambda_{1} \in \Lambda$ and $\Lambda \neq \emptyset$.

Lemma 3.3. Let (1.2) and (1.3) hold. If $\lambda_{0} \in \Lambda$, then $\lambda \in \Lambda$ for all $\lambda>\lambda_{0}$.

Proof. Let $\lambda_{0} \in \Lambda$ and $\lambda>\lambda_{0}$. Let $u_{\lambda_{0}}$ be a positive solution of $\left(1.1_{\lambda_{0}}\right)$. Then $u_{\lambda_{0}}$ is a supersolution of $\left(1.1_{\lambda}\right)$. We know that 0 is a subsolution of $\left(1.1_{\lambda}\right)$. By Theorem $2.1,\left(1.1_{\lambda}\right)$ has a solution $u_{\lambda_{1}}$ such that $0 \leq u_{\lambda} \leq u_{\lambda_{0}}$. By Proposition 2.8, $u_{\lambda}>0$ on $\bar{\Omega}$. Thus $\lambda \in \Lambda$.

Lemma 3.4. Let (1.2) and (1.3) hold. Then for every $\lambda>\lambda_{*}$, there exists a minimal positive solution $u_{\lambda}$ of $\left(1.1_{\lambda}\right)$ such that $u_{\lambda_{1}} \leq u_{\lambda_{2}}$ if $\lambda_{*}<\lambda_{2}<\lambda_{1}$.

Proof. Let $\lambda>\lambda_{*}$. Noting that 0 is a subsolution of $\left(1.1_{\lambda}\right)$ and 0 is not a solution of $\left(1.1_{\lambda}\right)$, we can see that there exists a minimal positive solution $u_{\lambda}$ of $\left(1.1_{\lambda}\right)$. Let $\lambda_{*}<\lambda_{2}<\lambda_{1}$ and let $u_{\lambda_{1}}$ and $u_{\lambda_{2}}$ are the minimal positive solutions of $\left(1.1_{\lambda_{1}}\right)$ and $\left(1.1_{\lambda_{2}}\right)$ respectively. Since $u_{\lambda_{2}}$ is a supersolution of $\left(1.1_{\lambda_{1}}\right)$, we can see that $u_{\lambda_{1}} \leq u_{\lambda_{2}}$

The proof of Theorem 1.1 is complete. Let us now turn to the proof of Theorem 1.2 .

Lemma 3.5. Let (1.2) and (1.3) hold. Let $\lambda_{1}, \lambda_{2} \in \Lambda$ and $\lambda_{2}<\lambda<\lambda_{1}$. Suppose that $u_{\lambda_{1}}$ and $u_{\lambda_{2}}$ are the positive solutions of $\left(1.1_{\lambda_{1}}\right)$ and $\left(1.1_{\lambda_{2}}\right)$ respectively and $u_{\lambda_{1}} \leq u_{\lambda_{2}}$. Then there exists a positive solution $v_{\lambda}$ of $\left(1.1_{\lambda}\right)$ such that $u_{\lambda_{1}} \leq$ $v_{\lambda} \leq u_{\lambda_{2}}$ and $v_{\lambda}$ is a global minimizer of the restriction of $J_{\lambda}$ to the order interval $\left[u_{\lambda_{1}}, u_{\lambda_{2}}\right] \cap W^{1, p(x)}(\Omega)$

Proof. Define $\tilde{f}: \bar{\Omega} \times \mathbf{R} \rightarrow \mathbf{R}$ by

$$
\tilde{f}(x, t)= \begin{cases}f\left(x, u_{\lambda_{1}}(x)\right), & \text { if } t<u_{\lambda_{1}}(x) \\ f(x, t), & \text { if } u_{\lambda_{1}}(x) \leq t \leq u_{\lambda_{2}}(x) \\ f\left(x, u_{\lambda_{2}}(x)\right), & \text { if } t>u_{\lambda_{2}}(x) .\end{cases}
$$


Define $\widetilde{F}(x, t)=\int_{0}^{t} \widetilde{f}(x, s) d s$ and for all $u \in W^{1, p(x)}(\Omega)$,

$$
\widetilde{J}_{\lambda}(u)=\int_{\Omega} \frac{|\nabla u|^{p(x)}+\lambda|u|^{p(x)}}{p(x)} d x-\int_{\partial \Omega} \varphi u d \sigma-\int_{\Omega} \widetilde{F}(x, u) d x .
$$

It is easy to see that the global minimum of $\widetilde{J}$ on $W^{1, p(x)}(\Omega)$ is achieved at some $v_{\lambda} \in W^{1, p(x)}(\Omega)$. Thus $v_{\lambda}$ is a solution of the following problem

$$
\left\{\begin{array}{l}
-\operatorname{div}\left(|\nabla u|^{p(x)-2} \nabla u\right)+\lambda|u|^{p(x)-2} u=\widetilde{f}(x, u) \quad \text { in } \Omega \\
|\nabla u|^{p(x)-2} \frac{\partial u}{\partial \eta}=\varphi \text { on } \partial \Omega,
\end{array}\right.
$$

and $v_{\lambda} \in C^{1}(\bar{\Omega})$. Noting that

$$
f\left(x, u_{\lambda_{1}}\right)=\widetilde{f}\left(x, u_{\lambda_{1}}\right) \leq \widetilde{f}\left(x, v_{\lambda}\right) \leq \widetilde{f}\left(x, u_{\lambda_{2}}\right)=f\left(x, u_{\lambda_{2}}\right)
$$

and $\lambda_{2}<\lambda<\lambda_{1}$, by Proposition 2.7.(4), we obtain that $u_{\lambda_{1}} \leq v_{\lambda} \leq u_{\lambda_{2}}$. So $\tilde{f}\left(x, v_{\lambda}\right)=f\left(x, v_{\lambda}\right)$, and $v_{\lambda}$ is a positive solution of $\left(1.1_{\lambda}\right)$. It is easy to see that there exists a constant $c$ such that $J_{\lambda}(u)=\widetilde{J}_{\lambda}(u)+c$ for $u \in\left[u_{\lambda_{1}}, u_{\lambda_{2}}\right] \cap W^{1, p(x)}(\Omega)$. Hence $v_{\lambda}$ is a global minimizer of $\left.J_{\lambda}\right|_{\left[u_{\lambda_{1}}, u_{\lambda_{2}}\right] \cap W^{1, p(x)}(\Omega)}$.

A key lemma of this paper is the following strong comparison principle.

Lemma 3.6 (A strong comparison principle). Let (1.2) and (1.3) hold. Let $\lambda_{1}, \lambda_{2} \in$ $\Lambda$ and $\lambda_{2}<\lambda_{1}$. Suppose that $u_{\lambda_{1}}$ and $u_{\lambda_{2}}$ are the positive solutions of $\left(1.1_{\lambda_{1}}\right)$ and $\left(1.1_{\lambda_{2}}\right.$ ) respectively and $u_{\lambda_{1}} \leq u_{\lambda_{2}}$. Then $u_{\lambda_{1}}<u_{\lambda_{2}}$ on $\bar{\Omega}$.

Proof. Since $u_{\lambda_{1}}, u_{\lambda_{2}} \in C^{1}(\bar{\Omega})$ and $u_{\lambda_{1}}>0$ on $\bar{\Omega}$, there exist the positive constants $b_{1} \leq 1$ and $b_{2}$ such that

$$
b_{1} \leq u_{\lambda_{1}} \leq u_{\lambda_{2}} \leq b_{2} \quad \text { on } \quad \bar{\Omega} .
$$

For $\varepsilon \in\left(0, \frac{b_{1}}{2}\right)$, setting $v_{\varepsilon}=u_{\lambda_{2}}-\varepsilon$, then

$$
\begin{aligned}
-\operatorname{div}\left(\left|\nabla v_{\varepsilon}\right|^{p(x)-2} \nabla v_{\varepsilon}\right)+\lambda_{1} v_{\varepsilon}^{p(x)-1}= & -\operatorname{div}\left(\left|\nabla u_{\lambda_{2}}\right|^{p(x)-2} \nabla u_{\lambda_{2}}\right)+\lambda_{2} v_{\varepsilon}^{p(x)-1} \\
& +\left(\lambda_{1}-\lambda_{2}\right) v_{\varepsilon}^{p(x)-1} \\
\geq & -\operatorname{div}\left(\left|\nabla u_{\lambda_{2}}\right|^{p(x)-2} \nabla u_{\lambda_{2}}\right)+\lambda_{2} u_{\lambda_{2}}^{p(x)-1} \\
& -\lambda_{2}\left(u_{\lambda_{2}}^{p(x)-1}-v_{\varepsilon}^{p(x)-1}\right) \\
& +\left(\lambda_{1}-\lambda_{2}\right)\left(\frac{b_{1}}{2}\right)^{p+-1} \\
= & f\left(x, u_{\lambda_{2}}\right)-\lambda_{2}\left(u_{\lambda_{2}}^{p(x)-1}-v_{\varepsilon}^{p(x)-1}\right) \\
& +\left(\lambda_{1}-\lambda_{2}\right)\left(\frac{b_{1}}{2}\right)^{p+1} .
\end{aligned}
$$

Taking an $\varepsilon>0$ sufficiently small such that

$$
\lambda_{2}\left(u_{\lambda_{2}}^{p(x)-1}-v_{\varepsilon}^{p(x)-1}\right)<\left(\lambda_{1}-\lambda_{2}\right)\left(\frac{b_{1}}{2}\right)^{p_{+}-1} \quad \text { for } \quad x \in \bar{\Omega},
$$


then

$$
-\operatorname{div}\left(\left|\nabla v_{\varepsilon}\right|^{p(x)-2} \nabla v_{\varepsilon}\right)+\lambda_{1} v_{\varepsilon}^{p(x)-1}=g(x) \geq f\left(x, u_{\lambda_{2}}\right),
$$

consequently, $v_{\varepsilon}$ is a solution of the problem

$$
\left\{\begin{array}{l}
-\operatorname{div}\left(|\nabla u|^{p(x)-2} \nabla u\right)+\lambda_{1}|u|^{p(x)-2} u=g(x) \text { in } \Omega \\
|\nabla u|^{p(x)-2} \frac{\partial u}{\partial \eta}=\varphi \text { on } \partial \Omega,
\end{array}\right.
$$

where $g(x) \geq f\left(x, u_{\lambda_{2}}\right)$. With other words, $v_{\varepsilon}=K_{\lambda_{1}}(g)$, where $K_{\lambda_{1}}$ is the solution operator of $\left(2.7_{\lambda_{1}}\right)$. Since $u_{\lambda_{1}}=K_{\lambda_{1}}(h)$, where $h(x)=f\left(x, u_{\lambda_{1}}\right) \leq f\left(x, u_{\lambda_{2}}\right) \leq$ $g(x)$, noting that $K_{\lambda_{1}}$ is increasing, we have $v_{\varepsilon} \geq u_{\lambda_{1}}$, that is, $u_{\lambda_{2}}-\varepsilon \geq u_{\lambda_{1}}$ on $\bar{\Omega}$.

Proof of Theorem 1.2. Let (1.2), (1.3) and (1.4) hold. Let $\lambda>\lambda_{*}$. Take $\lambda_{1}, \lambda_{2} \in \Lambda$ such that $\lambda_{2}<\lambda<\lambda_{1}$ and let $u_{\lambda_{1}} \leq v_{\lambda} \leq u_{\lambda_{2}}$ be as in Lemma 3.5. By Lemma 3.6, $u_{\lambda_{1}}<v_{\lambda}<u_{\lambda_{2}}$ on $\bar{\Omega}$. Thus there is a $C^{0}$-neighborhood $U$ of $v_{\lambda}$ such that $U \subset$ $\left[u_{\lambda_{1}}, u_{\lambda_{2}}\right]$, consequently $v_{\lambda}$ is a local minimizer of $J_{\lambda}$ in the $C^{0}$-topology, and of course, also in the $C^{1}$-topology. By Theorem 2.2, $v_{\lambda}$ is also a local minimizer of $J_{\lambda}$ in the $W^{1, p(x)}(\Omega)$-topology. Moreover, it is easy to see that, for $\lambda_{*}<\lambda_{2}<\lambda_{1}$, there are $u_{\lambda_{1}}$ and $u_{\lambda_{2}}$, being respectively a local minimizer of $J_{\lambda_{1}}$ and $J_{\lambda_{2}}$, such that $0<u_{\lambda_{1}} \leq u_{\lambda_{2}}$. Then, by Lemma 3.6, $u_{\lambda_{1}}<u_{\lambda_{2}}$ on $\bar{\Omega}$.

Remark 3.1. In [1] the Neumann problems $\left(1.1_{\lambda}\right)$ with $p(x) \equiv p$ have been considered. In the proof of Proposition 3.1 of [1], the authors of [1] think the assertion that $v_{\lambda}$ is a local minimizer of $J_{\lambda}$ in the $C^{1}$-topology can be obtained by the Proposition 2.1 in Guedda-Veron [24] (see [1, p. 1454]), this is a carelessness because the strong comparison principle established by Guedda-Veron [24] is appropriate for the Dirichlet problems but is not appropriate for the Neumann problems $\left(1.1_{\lambda}\right)$. Our Lemma 3.6 ensured the validity of the assertion of [1] Proposition 3.1.

Remark 3.2. It is easy to see that our Lemma 3.6 is also valid for the homogeneous Neumann boundary value problems.

Proof of Theorem 1.3. Let (1.2)-(1.5) hold. Let $\lambda>\lambda_{*}$. Take $\lambda_{1}, \lambda_{2} \in \Lambda$ such that $\lambda_{2}<\lambda<\lambda_{1}$ and let $u_{\lambda_{1}} \leq u_{\lambda} \leq u_{\lambda_{2}}$ be the above-mentioned positive solutions of $\left(1.1_{\lambda_{1}}\right),\left(1.1_{\lambda}\right)$ and $\left(1.1_{\lambda_{2}}\right)$ respectively and $u_{\lambda}$ a local minimizer of $J_{\lambda}$ in the $W^{1, p(x)}(\Omega)$-topology. Define

$$
\widetilde{f_{\lambda}}(x, t)= \begin{cases}f(x, t), & \text { if } t>u_{\lambda}(x), \\ f\left(x, u_{\lambda}(x)\right), & \text { if } t \leq u_{\lambda}(x)\end{cases}
$$

and $\widetilde{F}_{\lambda}(x, t)=\int_{0}^{t} \widetilde{f_{\lambda}}(x, s) d s$. Consider the problem

$$
\left\{\begin{array}{l}
-\operatorname{div}\left(|\nabla u|^{p(x)-2} \nabla u\right)+\lambda|u|^{p(x)-2} u=\widetilde{f}_{\lambda}(x, u) \quad \text { in } \Omega \\
|\nabla u|^{p(x)-2} \frac{\partial u}{\partial \eta}=\varphi \text { on } \partial \Omega,
\end{array}\right.
$$

and denote by $\widetilde{J}_{\lambda}$ the energy functional corresponding to $\left(3.4_{\lambda}\right)$. By the definition of $\tilde{f}_{\lambda}$, we have $\widetilde{f}_{\lambda}(x, u(x)) \geq f\left(x, u_{\lambda}(x)\right)$ for every $u \in W^{1, p(x)}(\Omega)$. Hence, for each 
solution $u$ of $\left(3.4_{\lambda}\right)$, we have that $u \geq u_{\lambda}$, consequently $\widetilde{f}_{\lambda}(x, u)=f(x, u)$ and $u$ is also a solution of $\left(1.1_{\lambda}\right)$. It is easy to see that $u_{\lambda_{1}}$ and $u_{\lambda_{2}}$ are a subsolution and a supersolution of $\left(3.4_{\lambda}\right)$ respectively. By Theorems 2.1 and 1.2, there exists $u_{\lambda}^{*} \in\left[u_{\lambda_{1}}, u_{\lambda_{2}}\right] \cap C^{1}(\bar{\Omega})$ such that $u_{\lambda}^{*}$ is a solution of $\left(3.4_{\lambda}\right)$ and is a local minimizer of $\widetilde{J}_{\lambda}$ in the $C^{1}$-topology. As was noted above, we know that $u_{\lambda}^{*} \geq u_{\lambda}$ and $u_{\lambda}^{*}$ is also a solution of $\left(1.1_{\lambda}\right)$. If $u_{\lambda}^{*} \neq u_{\lambda}$, then the assertion of Theorem 1.3 already holds, hence we can assume that $u_{\lambda}^{*}=u_{\lambda}$. Now $u_{\lambda}$ is a local minimizer of $\widetilde{J}_{\lambda}$ in the $C^{1}$-topology, and so also in the $W^{1, p(x)}(\Omega)$-topology. We can assume that $u_{\lambda}$ is a strictly local minimizer of $\widetilde{J}_{\lambda}$ in the $W^{1, p(x)}(\Omega)$-topology, otherwise we have obtained the assertion of Theorem 1.3. It is easy to verify that, under the assumptions of Theorem 1.3, $\widetilde{J}_{\lambda} \in C^{1}\left(W^{1, p(x)}(\Omega), \mathbf{R}\right)$ and $\widetilde{J}_{\lambda}$ satisfies the $(P . S)$ condition (see e.g. [19]). It follows from the condition (1.4) that $\inf \left\{\widetilde{J}_{\lambda}(u): u \in\right.$ $\left.W^{1, p(x)}(\Omega)\right\}=-\infty$. Using the mountain pass lemma (see [7]), we know that (3.4 $)$ has a solution $v_{\lambda}$ such that $v_{\lambda} \neq u_{\lambda} . v_{\lambda}$, as a solution of $\left(3.4_{\lambda}\right)$, must satisfy $v_{\lambda} \geq u_{\lambda}$, and $v_{\lambda}$ is also a solution of $\left(1.1_{\lambda}\right)$. The proof of Theorem 1.3 is complete.

Remark 3.3. In the proof of Theorem 1.3 the mountain pass lemma due to Ambrosetti and Rabinowitz [7] is used. For the applications of the mountain pass lemma to the Dirichlet problems with variable exponent we refer to $[16,17,19,32]$. In [19] and [16] the existence of a mountain pass type solution for the $p(x)$ Laplacian equation and the $p(x)$-Laplacian systems are obtained respectively. The common point between the main result of [32] and our Theorem 1.3 is that, using the mountain pass lemma for the energy functional $J$ having a positive minimizer $u$, it is proved that $J$ has the second positive critical point $v$ which is of mountain pass type. The big difference between the main result of [32] and our Theorem 1.3 is that, in the former the energy functional is coercive and $v \leq u$ but in the latter the energy functional is not coercive and $v \geq u$. The underlying ideas of the present paper are similar to that devoloped in [17], only in the present paper the inhomogeneous Neumann problems are considered and hence a special strong comparison principle, Lemma 3.6, is needed. As was mentioned in the introduction, Theorem 1.3 is a generalization of the corresponding result of [1] to the variable exponent case.

Proof of Theorem 1.4.(1). Let $f$ satisfy (1.2), (1.6) and (1.7). For given any $\lambda>$ 0 , consider the energy functional $J_{\lambda}$ defined by (2.9). By (1.7) and noting that $r(x)<p(x)$ for $x \in \bar{\Omega}$, there is a positive constant $M_{1}$ such that

$$
|F(x, t)| \leq \frac{\lambda}{2 p^{+}}|t|^{p(x)}, \quad \forall x \in \Omega, \quad \forall|t| \geq M_{1} .
$$

For $u \in W^{1, p(x)}(\Omega)$ with $\|u\|_{\lambda} \geq 1$, we have that

$$
\begin{aligned}
J_{\lambda}(u) \geq & \frac{1}{p^{+}} \int_{\Omega}\left(|\nabla u|^{p(x)}+\lambda|u|^{p(x)}\right) d x-\int_{\partial \Omega} \varphi u d \sigma \\
& -\frac{\lambda}{2 p^{+}} \int_{\Omega}|u|^{p(x)} d x-c_{1}
\end{aligned}
$$




$$
\begin{aligned}
& \geq \frac{1}{p^{+}} \int_{\Omega}|\nabla u|^{p(x)} d x+\frac{\lambda}{2 p^{+}} \int_{\Omega}|u|^{p(x)} d x-c_{2}\|u\|_{\lambda}-c_{1} \\
& \geq \frac{1}{2 p^{+}}\|u\|_{\lambda}^{p_{-}}-c_{2}\|u\|_{\lambda}-c_{1},
\end{aligned}
$$

where $c_{1}$ and $c_{2}$ are positive constants. This shows that $J_{\lambda}(u) \rightarrow+\infty$ as $\|u\|_{\lambda} \rightarrow$ $\infty$, that is, $J_{\lambda}$ is coercive. The condition (1.7) also implies that $J_{\lambda}$ is weakly sequentially lower semi-continuous. Thus $J_{\lambda}$ has a global minimizer $u_{0}$. Put $v_{0}(x)=$ $\left|u_{0}(x)\right|$ for $x \in \bar{\Omega}$. It is easy to see that $J_{\lambda}\left(v_{0}\right) \leq J_{\lambda}\left(u_{0}\right)$, consequently, $v_{0}$ is a global minimizer of $J_{\lambda}$ and is a positive solution of $\left(1.1_{\lambda}\right)$. This shows that $\lambda \in \Lambda$ for all $\lambda>0$. Hence $\lambda_{*}=0$ and the statement (1) is proved.

To prove Theorem 1.4.(2) we give the following two lemmata.

Lemma 3.7. If $f$ satisfies (1.2), (1.3) and there exists a positive constant $M_{1} \geq 1$ such that

$$
f(x, t) \geq t^{p(x)-1} \quad \text { for } \quad x \in \Omega \quad \text { and } \quad t \geq M_{1},
$$

Then

$$
\lambda_{*} \geq \min \left\{\frac{b}{M_{1}^{p_{+}-1}|\Omega|}, 1\right\},
$$

where $b=\int_{\partial \Omega} \varphi d \sigma$.

Proof. Let $\lambda \in \Lambda$ and $u$ be a positive solution of $\left(1.1_{\lambda}\right)$. Then, as was noted earlier, (3.1) holds, that is

$$
\lambda \int_{\Omega} u^{p(x)-1} d x-\int_{\Omega} f(x, u) d x=b .
$$

By the assumption for $\varphi, b>0$. To prove (3.7), arguing by contradiction, assume

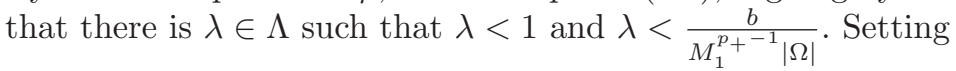

$$
\Omega_{1}=\left\{x \in \Omega: u(x)<M_{1}\right\} \quad \text { and } \quad \Omega_{2}=\left\{x \in \Omega: u(x) \geq M_{1}\right\}
$$

then

$$
\begin{aligned}
\lambda \int_{\Omega} u^{p(x)-1} d x-\int_{\Omega} f(x, u) d x & \leq \lambda \int_{\Omega_{1}} u^{p(x)-1} d x+\int_{\Omega_{2}} u^{p(x)-1} d x-\int_{\Omega_{2}} f(x, u) d x \\
& \leq \lambda \int_{\Omega_{1}} u^{p(x)-1} d x \leq \lambda M_{1}^{p_{+}-1}|\Omega|<b
\end{aligned}
$$

which contradicts $(3.8)$.

Remark 3.4. Obviously, (1.5) implies (3.6).

Lemma 3.8. Let (1.2) and (1.3) hold. Then for each $\lambda>\lambda_{*},\left(1.1_{\lambda}\right)$ has a positive solution $u_{\lambda}$ such that $J_{\lambda}\left(u_{\lambda}\right) \leq 0$. 
Proof. Let $\lambda>\lambda_{*}$. Take $\lambda_{2} \in\left(\lambda_{*}, \lambda\right)$ and let $u_{\lambda_{2}}$ be a positive solution of $\left(1.1_{\lambda_{2}}\right)$. then $u_{\lambda_{2}}$ is a supersolution of $\left(1.1_{\lambda}\right)$. We know that 0 is a subsolution of $\left(1.1_{\lambda}\right)$. Analogously to the proof of Lemma 3.5 , we can prove that $\left(1.1_{\lambda}\right)$ has a positive solution $u_{\lambda} \in\left[0, u_{\lambda_{2}}\right]$ such that $J_{\lambda}\left(u_{\lambda}\right)=\inf \left\{J_{\lambda}(u): u \in\left[0, u_{\lambda_{2}}\right]\right\}$. So $J_{\lambda}\left(u_{\lambda}\right) \leq$ $J_{\lambda}(0)=0$.

Remark 3.5. In fact, in Lemma 3.8 we have the assertion that $J_{\lambda}\left(u_{\lambda}\right)<0$. To see this, putting $v_{t} \equiv t$ on $\bar{\Omega}$, where $t$ is a positive constant, then for sufficiently small $t>0$, we have that $v_{t} \in\left[0, u_{\lambda_{2}}\right]$ and

$$
\begin{aligned}
J_{\lambda}\left(v_{t}\right) & =\int_{\Omega} \frac{\lambda t^{p(x)}}{p(x)} d x-\int_{\partial \Omega} \varphi t d \sigma-\int_{\Omega} F(x, t) d x \\
& \leq t^{p_{-}} \int_{\Omega} \frac{\lambda}{p(x)} d x-t \int_{\partial \Omega} \varphi d \sigma<0,
\end{aligned}
$$

thus $J_{\lambda}\left(u_{\lambda}\right) \leq J_{\lambda}\left(v_{t}\right)<0$.

Proof of Theorem 1.4.(2). Let (1.2)-(1.5) hold. By Lemma 3.7 and Remark 3.4, $\lambda_{*}>0$. Let $\lambda_{n}>\lambda_{*}$ and $\lambda_{n} \rightarrow \lambda_{*}$ as $n \rightarrow \infty$. By Lemma 3.8, for each $n,\left(1.1_{\lambda_{n}}\right)$ has a positive solution $u_{\lambda_{n}}$ such that $J_{\lambda_{n}}\left(u_{\lambda_{n}}\right) \leq 0$, that is

$$
\int_{\Omega} \frac{\left|\nabla u_{\lambda_{n}}\right|^{p(x)}+\lambda_{n}\left|u_{\lambda_{n}}\right|^{p(x)}}{p(x)} d x \leq \int_{\partial \Omega} \varphi u_{\lambda_{n}} d \sigma+\int_{\Omega} F\left(x, u_{\lambda_{n}}\right) d x .
$$

Since $u_{\lambda_{n}}$ is a solution of $\left(1.1_{\lambda_{n}}\right)$, we have that

$$
\int_{\Omega}\left(\left|\nabla u_{\lambda_{n}}\right|^{p(x)}+\lambda_{n}\left|u_{\lambda_{n}}\right|^{p(x)}\right) d x-\int_{\partial \Omega} \varphi u_{\lambda_{n}} d \sigma=\int_{\Omega} f\left(x, u_{\lambda_{n}}\right) u_{\lambda_{n}} d x .
$$

It follows from (1.5) that there exists a positive constant $c_{1}$ such that

$$
\int_{\Omega} F\left(x, u_{\lambda_{n}}\right) d x \leq c_{1}+\frac{1}{\theta} \int_{\Omega} f\left(x, u_{\lambda_{n}}\right) u_{\lambda_{n}} d x .
$$

Thus we have that

$$
\begin{aligned}
\frac{1}{p_{+}} \int_{\Omega}\left(\left|\nabla u_{\lambda_{n}}\right|^{p(x)}+\lambda_{n}\left|u_{\lambda_{n}}\right|^{p(x)}\right) d x \leq & \int_{\Omega} \frac{\left|\nabla u_{\lambda_{n}}\right|^{p(x)}+\lambda_{n}\left|u_{\lambda_{n}}\right|^{p(x)}}{p(x)} d x \\
\leq & \int_{\partial \Omega} \varphi u_{\lambda_{n}} d \sigma+c_{1}+\frac{1}{\theta} \int_{\Omega} f\left(x, u_{\lambda_{n}}\right) u_{\lambda_{n}} d x \\
\leq & \int_{\partial \Omega} \varphi u_{\lambda_{n}} d \sigma+c_{1}+\frac{1}{\theta} \int_{\Omega}\left(\left|\nabla u_{\lambda_{n}}\right|^{p(x)}\right. \\
& \left.+\lambda_{n}\left|u_{\lambda_{n}}\right|^{p(x)}\right) d x
\end{aligned}
$$

and consequently,

$$
\begin{aligned}
\left(\frac{1}{p_{+}}-\frac{1}{\theta}\right)\left\|u_{\lambda_{n}}\right\|_{\lambda_{n}}^{p_{-}} & \leq\left(\frac{1}{p_{+}}-\frac{1}{\theta}\right) \int_{\Omega}\left(\left|\nabla u_{\lambda_{n}}\right|^{p(x)}+\lambda_{n}\left|u_{\lambda_{n}}\right|^{p(x)}\right) d x+c_{2} \\
& \leq c_{3}\left\|u_{\lambda_{n}}\right\|_{\lambda_{n}}+c_{2}+c_{1},
\end{aligned}
$$

where the positive constants $c_{1}, c_{2}$ and $c_{3}$ are independent of $n$. This shows that $\left\{\left\|u_{\lambda_{n}}\right\|_{\lambda_{n}}\right\}$ is bounded. Noting that $\lambda_{n} \rightarrow \lambda_{*}>0$, we have that $\left\{\left\|u_{\lambda_{n}}\right\|\right\}$ is 
bounded. Without loss of generality we can assume that $u_{\lambda_{n}} \rightarrow u_{*}$ in $W^{1, p(x)}(\Omega)$ and $u_{\lambda_{n}}(x) \rightarrow u_{*}(x)$ for a.e. $x \in \Omega$. By (1.4) and the $L^{\infty}(\Omega)$-regularity results of [20], the boundedness of $\left\{\left\|u_{\lambda_{n}}\right\|\right\}$ implies the boundedness of $\left\{\left|u_{\lambda_{n}}\right|_{L^{\infty}(\Omega)}\right\}$. By the $C^{1, \alpha}(\bar{\Omega})$-regularity results of [18], the boundedness of $\left\{\left|u_{\lambda_{n}}\right|_{L^{\infty}(\Omega)}\right\}$ implies the boundedness of $\left\{\left\|u_{\lambda_{n}}\right\|_{C^{1, \alpha}(\bar{\Omega})}\right\}$, where $\alpha \in(0,1)$ is a constant. Thus we have $u_{\lambda_{n}} \rightarrow u_{*}$ in $C^{1}(\bar{\Omega})$. For every $v \in W^{1, p(x)}(\Omega)$, since $u_{\lambda_{n}}$ is a solution of $\left(1.1_{\lambda_{n}}\right)$, we have that, for each $n$,

$\int_{\Omega}\left(\left|\nabla u_{\lambda_{n}}\right|^{p(x)-2} \nabla u_{\lambda_{n}} \nabla v+\lambda_{n}\left|u_{\lambda_{n}}\right|^{p(x)-2} u_{\lambda_{n}} v\right) d x-\int_{\partial \Omega} \varphi v d \sigma=\int_{\Omega} f\left(x, u_{\lambda_{n}}\right) v d x$.

Passing the limit of above equality as $n \rightarrow \infty$, yields

$$
\int_{\Omega}\left(\left|\nabla u_{*}\right|^{p(x)-2} \nabla u_{*} \nabla v+\lambda_{*}\left|u_{*}\right|^{p(x)-2} u_{*} v\right) d x-\int_{\partial \Omega} \varphi v d \sigma=\int_{\Omega} f\left(x, u_{*}\right) v d x,
$$

which shows that $u_{*}$ is a solution of $\left(1.1_{\lambda_{*}}\right)$. Obviously $u_{*} \geq 0$ and $u_{*} \not \equiv 0$. Hence $u_{*}$ is a positive solution of $\left(1.1_{\lambda_{*}}\right)$ and $\lambda_{*} \in \Lambda$.

\section{Acknowledgements}

The author is grateful to the referee for the valuable comments and suggestions.

\section{References}

[1] E. A. M. Abreu, J. Marcos do Ó, E.S. Medeiros, Multiplicity of positive solutions for a class of quasilinear nonhomogeneous Neumann problems, Nonlinear Anal. 60 (2005), 1443-1471.

[2] E. Acerbi, G. Mingione, Regularity results for a class of functionals with nonstandard growth, Arch. Ration. Mech. Anal. 156 (2001), 121-140.

[3] R. A. Adams, Sobolev Spaces, Academic Press, New York, 1975.

[4] C. O. Alves, M.A.S. Souto, Existence of solutions for a class of problems in $R^{N}$ involving the $p(x)$-Laplacian, Progress in Nonlinear Differential Equations and Their Applications 66 (2005), 17-32.

[5] H. Amann, Fixed point equations and nonlinear eigenvalue problems in ordered Banach spaces, SIAM Remview 18 (1976), 620-709.

[6] A. Ambrosetti, H. Brezis, G. Cerami, Combined effects of concave and convex nonlinearities in some elliptic problems, J. Funct. Anal. 122 (1994), 519-543.

[7] A. Ambrosetti, P. Rabinowitz, Dual variational methods in critical point theory and applications, J. Funct. Anal. 14 (1973), 349-381.

[8] J. G. Azorero, J. J. Manfredi, I. P. Alonso, Sobolev versus Hölder local minimizer and global multiplicity for some quasilinear elliptic equations, Comm. Contemp. Math. 2 (2000), 385-404.

[9] H. Brezis, L. Nirenberg, $H^{1}$ versus $C^{1}$ local minimizers, C. R. Acad. Sci. Paris Ser. I Math. 317 (1993), 465-472.

[10] K. C. Chang, A variant of mountain pass lemma, Scientia Sinica Ser. A 26 (1983), $1241-1255$. 
[11] L. Damascelli, B. Sciunzi, Harnack inequalities, maximum and comparison principles, and regularity of positive solutions of m-Laplace equations, Calc. Var. PDE. 25 (2005), 139-159.

[12] L. Damascelli, B. Sciunzi, Regularity, monotonicity and symmetry of positive solutions of $m$-Laplace equations, J. Differential Equations 206 (2004), 483-515.

[13] Y.B. Deng, S. J. Peng, Existence of multiple positive solutions for inhomogeneous Neumann problem, J. Math. Anal. Appl. 271 (2002), 155-174.

[14] L. Diening, P. Hästö, A. Nekvinda, Open problems in variable exponent Lebesgue and Sobolev spaces, FSDONA04 Proceedings, (P. Drábek and J. Rákosník (eds)), Milovy, Czech Republic, 38-58, 2004.

[15] D. E. Edmunds, J. Rákosník, Sobolev embedding with variable exponent, Studia Math. 143 (2000), 267-293.

[16] A. El Hamidi, Existence results to elliptic systems with nonstandard growth conditions, J. Math. Anal. Appl. 300 (2004), 30-42.

[17] X. L. Fan, On the sub-supersolution method for $p(x)$-Laplacian equations, J. Math. Anal. Appl. 330 (2007), 665-682.

[18] X. L. Fan, Global $C^{1, \alpha}$ regularity for variable exponent elliptic equations in divergence form, J. Differential Equations 235 (2007), 397-417.

[19] X. L. Fan, Q. H. Zhang, Existence of solutions for $p(x)$-Laplacian Dirichlet problems, Nonlinear Anal. 52 (2003), 1843-1852.

[20] X. L. Fan, D. Zhao, A class of De Giorgi type and Hölder contihuity, Nonlinear Anal. 36 (1996), 295-318.

[21] X. L. Fan, D. Zhao, On the spaces $L^{p(x)}(\Omega)$ and $W^{m, p(x)}(\Omega)$, J. Math. Anal. Appl. 263 (2001), 424-446.

[22] X. L. Fan, Y.Z. Zhao, Q.H. Zhang, A strong maximun principle for $p(x)$-Laplace equations, Chinese J. Contemp. Math. 24(3) (2003), 277-282.

[23] D. Gilbarg, N.S. Trudinger, Elliptic partial differential equations of second order, 2nd ed., Springer Verlag, Berlin, 1983.

[24] M. Guedda, L. Veron, Quasilinear elliptic equations involving critical Sobolev exponents, Nonlinear Anal. 13 (1989), 879-902.

[25] Z. M. Guo, Z. T. Zhang, $W^{1, p}$ versus $C^{1}$ local minimizers and multiplicity results for quasilinear elliptic equations, J. Math. Anal. Appl. 286 (2003), 32-50.

[26] Y. Il'yasov, T. Runst, On nonlocal calculation for inhomogeneous indefinite Neumann boundary value problems, Calc. Var. PDE. 22 (2005), 101-127.

[27] O. Kováčik, J. Rákosník, On spaces $L^{p(x)}(\Omega)$ and $W^{k, p(x)}(\Omega)$, Czechoslovak Math. J. 41(116) (1991), 592-618.

[28] V.K. Le, K. Schmitt, quasilinear elliptic equations and inequalities with rapidly growing coefficients, J. London Math. Soc. 62 (2000), 852-872.

[29] G. M. Lieberman, Mixed boundary value problems for elliptic and parabolic differential equations of second order, J. Math. Anal. Appl. 113 (1986), 422-440.

[30] G. M. Lieberman, Boundary regularity for solutions of degenerate elliptic equations, Nonlinear Anal. 12 (1988), 1203-1219.

[31] P. Marcellini, Regularity and existence of solutions of elliptic equations with $(p, q)$ growth conditions, J. Differential Equations 90 (1991), 1-30. 
[32] M. Mihăilescu, V. Rădulescu, A mulyiplicity result for a nonlinear degenerate problem arising in the theory of electrorheological fluids, Proc. Royal Soc. A 462 (2006), $2625-2641$.

[33] M. Mihăilescu, V. Rădulescu, On a nonhomogeneous quasilinear eigenvalue problem in Sobolev spaces with variable exponent, Proc. Amer. Math. Soc. 135 (2007), 29292937.

[34] X. B. Pan, Futher study on the effect of boundary conditions, J. Differential Equations 117 (1995), 446-468.

[35] S. I. Pohozaev, A. Tesei, Existence of positive solutions to some nonlinear Neumann problems, Doklady Mathematics 58 (1998) 414-417. Translated from Doklady Akademii Nauk 363 (1998), 450-453.

[36] P. Pucci , J. Serrin, The strong maximum principle revisited, J. Differential Equations 196 (2004), 1-66.

[37] M. Růžička, Electrorheological Fluids: Modeling and Mathematical Theory, Lecture Notes in Math., Vol. 1748, Springer Verlag, Berlin, 2000.

[38] S. Samko, On a progress in the theory of Lebesgue spaces with variable exponent: maximal and singular operators, Integral Transforms and Special Functions 16 (2005), 461-482.

[39] S. Samko, B. Vakulov, Weighted Sobolev theorem with variable exponent for spatial and spherical potential operators, J. Math. Anal. Appl. 310 (2005), 229-246.

[40] A. Siai, Nonlinear Neumann problems on bounded Lipschitz domains, Ellictronic J. Differential Equations 2005 (2005), No. 09, 1-16.

[41] V. V. Zhikov, On some variational problems, Russian J. Math. Phys. 5 (1997), 105116.

[42] V. V. Zhikov (=V. V. Jikov), S. M. Kozlov, O. A. Oleinik, (Translated from the Russian by G. A. Yosifian), Homogenization of Differential Operators and Integral Functionals, Springer Verlag, Berlin, 1994.

Xianling Fan and Shao-Gao Deng

Department of Mathematics

Lanzhou University

Lanzhou 730000

China

e-mail: fanxl@lzu.edu.cn (X-L Fan)

dengshg03@lzu.cn (S-G Deng)

Submitted: 14 September 2006.

Revised: 9 October 2008.

Accepted: 25 October 2008. 